\title{
Enseñanza de la música contemporánea a través de nuevas tecnologías
}

\author{
Una experiencia pedagógica en el aula universitaria
}

\author{
Marta Vela González' \\ marta.vela@unir.net \\ Universidad Internacional de La Rioja
}

\section{Resumen}

La recepción de la música contemporánea continúa siendo, en la actualidad, un tema espinoso, a nivel mundial, dado el desencuentro de este tipo de música con el público. Se puede apreciar una situación parecida a nivel educativo, en que la música de los siglos XX y XXI generalmente se rehúye a nivel docente y discente. El presente estudio pretende transmitir una experiencia didáctica en estudiantes de nivel superior, en que el proceso de enseñanzaaprendizaje de la música contemporánea se ha tratado con técnicas innovadoras, desde un modelo educativo a distancia, $100 \%$ on line. Con una muestra de estudiantes sin experiencia previa en la materia, matriculados en la asignatura optativa de Análisis de la música de los siglos XX y XXI, se ha tratado de elucidar el grado de interés del alumnado, así como el grado de conocimiento obtenido en función de los resultados, en que han incidido cuatro factores de aprendizaje concretos. El uso de recursos digitales, a través del campus virtual, así como la práctica de análisis musical gráfico-sonoro, se ha traducido en unos rendimientos finales exitosos.

\section{Palabras Clave}

Educación on line, análisis, música contemporánea, enseñanza, estrategias innovadoras. 


\title{
Teaching of contemporary music through new technologies
}

\author{
An education experience in university classroom
}

\section{Abstract}

The reception of contemporary music continues to be, at present, for several decades, a thorny issue, worldwide, given the disagreement of this type of music with the public. You can see a similar situation at the educational level, in which the music of the twentieth and twenty-first, generally, shies away from teaching and learning. The present study intends to transmit a didactic experience in higher level students, in which the teaching-learning process of contemporary music has been treated with innovative techniques, from a distance educational model, $100 \%$ on line. With a sample of students with no previous experience in the subject, enrolled in the optional subject of Analysis of music of the XX and XXI centuries, it has been tried to elucidate the degree of interest of the students, as well as the degree of knowledge obtained in function of the results, in which four very specific learning factors have affected. The use of digital resources, through the virtual campus, as well as the practice of graphic-sound music analysis, has resulted in successful final performances.

\section{Key Words}

On line education, analysis, contemporary music, teaching, innovative strategies.

\section{Introducción}

En primer lugar, definiremos el concepto de música contemporánea dentro del panorama actual de la música académica, siguiendo a Harnoncourt (2006), elucidada como música seria: "si pensamos en la música actual, observamos enseguida que está dividida: diferenciamos entre música popular, música ligera y música seria (...) Dentro de cada una de estas categorías se pueden encontrar elementos de unidad" (p. 22). Se observan, en suma, tres niveles separados, la música popular -que también podría incluir a la música tradicional, a la que podría unirse la música étnica, tan de boga en este momento-; la música ligera o comercial, aquélla derivada de los estilos pop y rock del siglo XX, procedentes de la música negra norteamericana; y, finalmente, la música seria, culta, clásica, teniendo en cuenta que este último término, altamente popularizado en los últimos años, sobre todo, para los profanos, comprende, en realidad, sólo la música de un período artístico muy delimitado en el tiempo, al que pertenece la música de Haydn, Mozart y Beethoven. 
La perspectiva global de la música seria puede, a su vez, descomponerse de la siguiente manera, en tres apartados temporales entre sí:

1. La música antigua: que comprende el Barroco y el Renacimiento, anterior, por tanto, a 1750.

2. La música histórica: que se ubica en una posición temporal intermedia de los dos externos: 1750 y 1900.

3. La música contemporánea: la música seria creada en nuestros días, que abarca, en realidad, toda la música del pasado siglo XX y hasta la actualidad.

A continuación, se expone la problemática de esta llamada música contemporánea, dado que su situación actual plantea, ya, de inicio, una paradoja, puesto que, en tanto que contemporánea, se debería suponer simultánea a la actualidad y, sin embargo, delimita toda aquélla música, desde el inicio del siglo XX, que renuncia explícitamente al sistema tonal -tal cual se había conocido durante los siglos XVII, XVIII y XIX-y, posteriormente, a la idea de textura y forma tradicional que aún conservan la música de Schönberg y las tendencias modernistas hasta 1945.

De esta paradoja se deduce la dificultad de la música contemporánea de la actualidad y, por ende, la de su enseñanza, porque la música compuesta hace cien años debería tener la consideración de música histórica (Harnoncourt, 2006), según el devenir del tiempo y, por tanto, de ningún modo debería considerarse contemporánea a nosotros: no lo es.

De esta forma, el término contemporáneo resulta ambiguo, erróneo, si se quiere, porque no denota una cualidad temporal, como cabría esperarse, sino una premisa estética, a saber, la condición atonal de la música, que se ha extendido durante más de cien años en un mismo medio artístico, que empezó con los llamados movimientos históricos de vanguardia, como lo ha definido Bürger:

Evidentemente, el medio artístico es la categoría general para la descripción de las obras de arte. Pero los procedimientos particulares sólo pueden ser conocidos como medio artístico desde los movimientos históricos de vanguardia. Únicamente en los movimientos históricos de vanguardia se hace disponible como medio la totalidad del medio artístico (Bürger, 1987: 56).

En la actualidad, probablemente, ya disponemos de la suficiente "perspectiva histórica" que demandaba Cureses (1998), una vez finalizado el azaroso siglo XX, para abordar la reflexión y, por tanto, la organización del proceso de enseñanza- 
aprendizaje de la música contemporánea, sobre todo, a nivel universitario, a la que anteponemos el siguiente apartado de trayectoria histórica.

\section{La música contemporánea hasta la actualidad}

Convendremos en que la música contemporánea -la nueva música (Adorno, 2000; Webern, 2009) - ha sido fuente de conflictos y polémicas desde el momento mismo de su aparición (Schoenberg, 1988; Lagrange, 2002; Buch, 2010), en un entorno, la Viena de la posguerra, especialmente conservador en materia musical, que no supo recibir una música disonante, elaborada al margen de la tonalidad y, por tanto, alejada de la dimensión de consonancia observada en el origen físicoacústico de la música, la serie de armónicos naturales.

El Dodecafonismo acabó con la tonalidad y abrió una nueva era, extraña e incierta, del mismo modo que la guerra habría arrasado el continente y algunas de sus instituciones centenarias, en el caso, por ejemplo, de la monarquía austríaca. Esta tendencia, como otras vanguardias de la época, surgió de una sociedad convulsa y errática, en un contexto artístico expresionista y, de este modo, obligó al creador a un posicionamiento estético, probablemente, inédito hasta ese momento, en un mundo fuertemente polarizado. En efecto, durante aquellos años, la música, al igual que otras ramas del arte, adquirió una función reivindicativa -política, social, nacionalista, etc.- y perdió, entonces, su antigua dimensión estética, burguesa, asociada al placer:

En un mundo atroz, el arte debe demoler el concepto de goce con el que tradicionalmente se le asociaba. Legítimamente ya no puede producir placer ni deleitar. La música de Schönberg es auténtica porque tanto por su forma como por su contenido se mide sin reparos con la experiencia del espanto. Esa música se hizo inhumana hasta sobrepasar la inhumanidad del mundo por amor del hombre (Adorno, 2000, p. 14).

En efecto, la música de Schönberg causó numerosos escándalos en época de entreguerras y, de hecho, su música, junto a la de Werbern -Berg había muerto en 1935- fue calificada como entartete, degenerada, por el régimen nazionalsocialista, a pesar de que los integrantes de la llamada Segunda Escuela Vienesa -esta denominación resulta toda una declaración de intenciones en este sentido, dado que nos remite, directamente, al período clásico de Haydn, Mozart y Beethoven- componían en el profundo convencimiento de que su música suponía la evolución natural de la música alemana, a pesar del abandono del sistema tradicional: "la pérdida de la tonalidad resultó sumamente perturbadora por más que, 
retrospectivamente, la historia del arte musical del siglo XIX pareciera conducir ineludiblemente a ella", (Rosen, 2017, p. 12).

Ante las críticas, los compositores de la Nueva Música siempre afirmaron que sus creaciones no eran apreciadas porque no se entendían y que el tiempo, en efecto, pondría a cada uno en su lugar. Éste fue el primer argumento de Schönberg y sus alumnos, en la apasionada defensa de su arte: "la serie de los armónicos hay que definirla como prácticamente infinita. Siempre es posible imaginar nuevas diferenciaciones cada vez más sutiles (...) la única cuestión es saber si la época actual está madura para ello" (Webern, 2009, p. 31); "pienso que sólo puede argüirse contra mí que escribo una música que no gusta a aquéllos que no entienden nada de ella. A la par que se puede añadir que gusta a aquéllos que entienden" (Schoenberg, 1988, p. 28). Pese a todo, otros compositores de la época, formados, al igual que Schönberg y los suyos, en el último romanticismo, fueron incapaces de convertirse y abrazar las nuevas corrientes, por lo que fueron tachados, automáticamente, de reaccionarios: "«Me siento como un fantasma vagando en un mundo que se ha vuelto extraño -escribía Rachmaninov en 1939-. No puedo desterrar el antiguo modo de escribir, y no soy capaz de adquirir el nuevo. Me he esforzado enormemente por sentir las maneras musicales actuales, pero no van a acudir a mí” (Ross, 2010, p. 207).

Sin embargo, pronto surgieron voces críticas hacia la Nueva Música que, frente a la opinión de los dodecafónicos, no provenían de un público ignorante, incapaz de comprender las técnicas compositivas más avanzadas, sino de intérpretes respetables y más que consagrados, perfectamente capaces de deleitarse con los artificios compositivos de las nuevas tendencias, como Walter y Furtwängler, ambos muy críticos con la deriva atonal de la música durante las primeras décadas del siglo XX:

Los efectos que han podido tener dos guerras mundiales y su prolongada duración en el pensamiento, las emociones y el comportamiento de nuestros contemporáneos, y los daños que han causado al espíritu y al contenido de nuestra cultura han sido tan espantosos, que es alentador constatar que una obra [la de Mahler] característica de una edad de la razón, a pesar de su originalidad, madurada bajo la influencia de los grandes clásicos, ha llegado a ocupar semejante lugar en el seno de nuestro turbado mundo. Basta considerar, por ejemplo, las dramáticas consecuencias de la crisis que siguió a la Primera Guerra Mundial; el arte, unas veces intelectualizado, otras, degenerado en pura y simple diversión, de la idolatría de las proezas técnicas, del atonalismo, del sistema dodecafónico, de las experiencias musicales más gratuitas, todo en un clima de materialismo y de conflicto ideológico cada vez más violento (Walter, 2002, p. 38). 
Es asombroso a veces el derroche de inteligencia que se encuentra en las múltiples combinaciones de la música atonal. El logro del intelecto puede, en ciertas circunstancias, elevarse a alturas extraordinarias. Pero el precio que tiene que pagar, desde el punto de vista biológico, es la falta de valores vitales (...) No podemos cerrarnos a la evidencia de que una música prescinde de la capacidad para regular la tensión y la relajación, sacrificando así la precisión geográfica de la tonalidad -cuales quiera que sean sus otras cualidades- debe ser considerada biológicamente inferior (Furtwängler, 2011, pp. 99-100).

A pesar de las críticas, la Nueva Música continuó en desarrollo a partir del fin de la Segunda Guerra Mundial y, de hecho, se vio revitalizada en los Cursos de Darmstadt, desde 1946, a partir de un nuevo modelo compositivo, una versión alambicada del estilo dodecafónico desde el ejemplo de las obras de Webern apreciada tempranamente, por ejemplo, en Mode de valeurs et d' intensités de Messiaen (1949)-, el Serialismo Integral, cuya rigidez y dogmatismo desposeyeron a la música de cualquier resquicio expresivo, sobre todo, durante las décadas de los años cincuenta y sesenta. La fiereza con que los compositores defendieron la práctica serial -fundamentalmente, Boulez y Stockhausen, los dos enfants terribles de la música de posguerra, "los tiranuelos más exclusivamente terroristas de la joven música, falsamente deducida del estilo de Webern", en la opinión de Walter (2002, p. 141) - les hizo ahogar todo tipo de música contemporánea contraria a sus preceptos estéticos durante décadas, a la que reaccionaron, desde finales de los cincuenta, no sólo tendencias consonantes como Minimalismo y Nueva Simplicidad, sino también, líneas extremadamente disonantes, por ejemplo, la música de textura (Xenakis, 1955) y, en general, buena parte del mundo musical que ansiaba el regreso a la música decimonónica: "así, de acuerdo con un saber sacado de las obras de sus propios predecesores, se niega al artista toda soberanía, libertad y espontaneidad de expresión, se le prescribe lo que debe hacer y lo que no, lo que debe sentir y querer para poder contar con tener repercusión, para ser moderno" (Furtwängler, 2011, p. 77).

Desde la posguerra hasta la actualidad, la música contemporánea se ha diversificado en un sinfín de tendencias, muy influenciada, además, por las tendencias electroacústicas, en lo que podríamos definir como un segundo fin de siècle (Ross, 2010) en que predomina la combinación ecléctica de diversos estilos y el maridaje de lo acústico con lo electrónico, es decir, un variopinto crisol, como ha declarado Ros Marbà, cuyo gusto por la continua renovación del lenguaje musical se ha vuelto cargante: "el peligro del concepto de evolución constante que rige en numerosas composiciones contemporáneas, en las que se evitan reiteraciones y se proponen constantes novedades, es que quede eliminado cualquier punto de referencia. La variedad misma se vuelve monótona" (Madigan, 2008, p. 28). 
Así lo ha observado también Bürger (1987, p. 56), en cuanto a la ruptura del concepto de "estilo":

Hasta ese momento del desarrollo del arte, la aplicación del medio artístico estaba limitada por el estilo de la época, un canon de procedimientos admisibles infringido sólo aparentemente, dentro de unos límites estrechos. Mientras el estilo domina, la categoría de medio artístico es opaca porque, en realidad, sólo se da como algo especial. Un rasgo característico de los movimientos históricos de vanguardia consiste, precisamente, en que no han desarrollado ningún estilo; no hay un estilo dadaísta ni un estilo surrealista. Estos movimientos han acabado, más bien, con la posibilidad de un estilo de la época, ya que han convertido en principio la disponibilidad de un estilo de la época, ya que han convertido en principio la disponibilidad de los medios artísticos de las épocas pasadas. Sólo la disponibilidad universal hace general la categoría de medio artístico.

De hecho, algunos autores como Ansermet (2000) se preguntaban hasta qué punto era necesaria una renovación que, en realidad, deconstruía el propio lenguaje musical -el de la tonalidad- hasta hacerlo irreconocible: "¿Es decir que el lenguaje de la música no podía renovarse? ¡Seguro que no! Cuando un lenguaje -el francés, el alemán, etcétera- ha culminado la constitución de sus estructuras, a saber, su sintaxis y su vocabulario fundamental, la creación literaria puede proseguir indefinidamente" (p. 141-142).

De esta manera, por su clara voluntad de ruptura con la tonalidad y, posteriormente, con el resto de parámetros de la música del pasado -textura, forma, armonía- la música contemporánea se ha alejado paulatinamente, como propugnaba Adorno, de la idea tradicional de la conexión con las emociones humanas, el afecto, el ethos, si se quiere -tal cual se conocieron en la música de los siglos anteriores- y, por otra parte, su insistencia en renovar el lenguaje musical de manera constante, tampoco ha conseguido ofrecer al oyente un estilo unitario en que el público pueda reconocerse, mostrando, en efecto, una sensación de desconcierto y ruido, como explicaba Casals: "la música contemporánea -mejor dicho, la «música caótica»- presenta una lamentable tendencia a excluir todo cuanto sea humano y natural, y, sobre todo, lo que podría ser pura y auténtica emoción. ¿Qué comunicación con los demás puede establecerse partiendo de tal actitud?" (Corredor, 1985: 115). En consecuencia, con la excesiva intelectualización de sus propuestas y su continua novedad en el estilo -además de su universo sonoro, manifiestamente disonante-, la música contemporánea ha conseguido que el público -salvo cierto sector especializado, perteneciente, en su mayoría, a la misma génesis de la música contemporánea, esto es, compositores e intérpretes de música contemporánea- se aleje por sistema del repertorio contemporáneo, porque buena parte de los com- 
positores se han visto en la obligación de generar música sólo interesante para el escalón más elevado de la academia: "el público general que escucha música quisiera descubrir aun algo y hallar tranquilidad y recogimiento en las relaciones tonales. El propio Bartók solía asegurar que jamás había abandonado la tonalidad" (Menuhin, 2005, p. 111).

En la actualidad, el oyente medio -incluso, es incapaz de nombrar alguna de las figuras de referencia en música contemporánea -Boulez, Stockhausen, Berio, Ligeti-, ni una obra de referencia desde 1945. De hecho, en muchos círculos musicales de la actualidad se ignora la existencia de los Cursos de Darmstadt y su importancia en el desarrollo de la música académica durante la segunda mitad del siglo XX: podría decirse que la música contemporánea es un fenómeno completamente ajeno al público de la actualidad, que prefiere consumir una música culta más sencilla y aprehensible, del estilo que sea.

La modernidad en pintura y literatura se soporta más fácilmente que en música, ya que uno puede pasar de largo rápidamente junto a la pintura o cerrar el libro. Pero sentarse pacientemente durante una interpretación pública de una obra musical larga y moderna es una tortura para el oyente adverso, y no es sorprendente que ocasionalmente inspire perturbadoras salidas calculadas para arruinar el placer de los que se quedan (Rosen, 2014, pp. 241-242).

Esta perspectiva ha obligado a revisar las opiniones de los más férreos defensores del género: "cuando le preguntaron [a Boulez] en 1999 por qué tan pocas obras importantes de los años cincuenta y sesenta habían entrado a formar parte del repertorio, confesó sin inmutarse: «Bueno, quizá no tuvimos suficientemente en cuenta el modo en que la música es percibida por el oyente»" (Ross, 2010, p. 646).

Así pues, la popularización de la música contemporánea, que Schönberg predijo desde sus inicios, ni ha llegado ni llegará: esta circunstancia responde a la problemática de la difusión y enseñanza de la música contemporánea, que analizaremos a continuación. De nuevo, encontramos una paradoja: aunque el público acogió con reservas buena parte de la música de Beethoven -sobre todo, la del período intermedio, la del período final apenas fue interpretada en vida del autor-, rápidamente, en menos de veinte años tras su muerte, se revalorizó como paradigma artístico en todo el mundo, y, por el contrario, la Nueva Música, que pronto cumplirá un siglo de vida, no ha conquistado al público ni a los programadores de las salas de concierto, incluso, la misma vanguardia, acusó al mismo Schönberg de reaccionario ${ }^{1}$ tras su muerte. 


\section{La enseñanza de la música contemporánea}

Sin duda, uno de los objetivos de la enseñanza de la música contemporánea, todavía, reside en el derribo de los distintos prejuicios que hasta hoy mismo la rodean, como confesaba Boulez (2008, p. 17): "entre los aficionados a la cultura, hay muchos que no desean cambiar sus hábitos, lo sabemos muy bien". De hecho, sólo a través de la formación en la música de nuestros días puede lograrse el acercamiento, cada vez más temprano, de nuevos públicos. Según Hemsy de Gainza (2002), la música contemporánea empieza a incluirse en la enseñanza a mediados de los años setenta del siglo XX, con los "aportes de George Self y Brian Dennis en Inglaterra, Lili Friedmann en Alemania, Floke Rabe y Jan Bark en Suecia, y el Contemporary Music Project en los Estados Unidos", a los que se podría añadir "sobre todo, las diferentes líneas de investigación y manipulación sonora posteriores al dodecafonismo de la Escuela de Viena", junto a otros autores como Murray Schafer, Francois Delalande, Guy Reibel o John Paynter.

Probablemente, la enseñanza de la música contemporánea ha chocado, desde el principio, con la poca fe que los propios compositores de vanguardia albergaban sobre sus efectos: "la pedagogía no es la panacea. Quiero decir que no va a dar talento a quien no lo tiene", (Boulez, 2002, p. 157), o "no es fácil desperdiciar nuestro tiempo en adoctrinar alumnos absolutamente negados para la música. Sería algo así como aceptar la esclavitud como moneda de cambio para la independencia del mañana, de la independencia artística, naturalmente", (Stockhausen, 1998, p. 87).

De este modo, tanto Boulez como Stockhausen, dos de las figuras más representativas de la llamada Neue Musik, de los años de la posguerra, pese a su paso por distintas instituciones educativas de nivel superior en su juventud, por ejemplo, el Conservatorio de París, donde asistieron a las clases de Messiaen, entre otros, abogaban por un aprendizaje autodidacta: "la pedagogía no es sino un principio; lo realmente importante es enseñarse a uno mismo. Lo he dicho más de una vez y lo sostengo: prefiero a los autodidactas por voluntad que a los autodidactas por azar", Boulez, 2002, pp. 158-159). Stockhausen, por su parte, apostaba por un modelo pedagógico tradicional, maestro-discípulo, perteneciente a la enseñanza de la música en el pasado, antes, incluso, de la aparición de los conservatorios y de las universidades de música, es decir, anterior a 1800:

El lenguaje de la música contemporánea se agota rápidamente, ya que cada obra busca y asume el suyo propio. Hay un imperativo: renovarse o buscar lo inaudito o lo inédito. Antes se solía escribir lo que se solía oír, bastaba algo de talento y atención y un cuidadoso estudio del trabajo ajeno para componer a los dieciocho años. Hoy sería impensable: finalmente, ha vencido la creatividad. La música se ha 
emancipado, la música culta ha dejado de ser un entretenimiento. Por ello -repitono existe más solución didáctica que la de organizarse un aprendizaje propio, junto a un gran maestro (Stockhausen, 1998, p. 89).

Sin embargo, todo acercamiento a la música contemporánea y a su composición parte de la premisa del análisis de la música anterior: "creo que no se puede enseñar la composición por sí misma, salvo si se recurre al análisis de partituras para mostrar las vías" (Boulez, 2002, p. 152), pero, según Boulez, desde un particular enfoque analítico, lejos de las dimensiones, tal vez, más intelectualizadas del siglo XX, como la fenomenología o la estadística, que perduran en la actualidad:

Hemos asistido, aquí y allá, a un florecimiento exuberante de análisis más o menos absurdos que, bajo diversos pretextos -fenomenología, estadística- se han degradado abocando a deplorables criaturas. Hasta hace mucho poco, los análisis contables llegaban casi a desconsiderar el objeto que se proponían como fin de un estudio exhaustivo; y esto es precisamente lo que pasa, más recientemente, en estas investigaciones que, combinando estadística e información, se reducen a contar los frutos de un árbol, o a describirlos sin tener en cuenta el árbol mismo e ignorando impávidamente el proceso de fecundación (Boulez, 2009, p. 30).

Así pues, frente al tradicional análisis paramétrico -estilo, forma, armonía, etc.,-, los compositores de la segunda mitad del siglo XX fijaron nuevas concepciones estructurales en virtud del material sonoro de su música, marcada, fundamentalmente, por la textura y el timbre, con un proceso de parametrización basado en dos únicos elementos, la estructura y el proceso, dos fenómenos que abarcan la totalidad de la música contemporánea, como reconocieron en los años sesenta, respectivamente, dos figuras de la vanguardia, Cage, The Future of Music: Credo (1937) y Boulez, en Penser la musique aujourd'bui (1963).

Para concluir, podríamos definir lo que consideramos como los componentes indispensables de un método analítico activo: se debe partir de una observación tan exacta y minuciosa como sea posible de los hechos musicales que se nos propone; se trata, a continuación, de encontrar un esquema, una ley de organización interna que dé cuenta, con el máximo de coherencia, de estos hechos; finalmente, viene la interpretación de las leyes de composición deducidas de esta aplicación particular. Todas estas etapas son necesarias. El no llegar hasta la etapa principal: la interpretación de las estructuras es quedarse en un trabajo técnico completamente secundario (Boulez, 2009, pp. 32-33). 
Muchos compositores ya no componen estructuras. En lugar de ello, ponen procesos en marcha. Una estructura es como un mueble, mientras que un proceso es como el tiempo meteorológico (...) desde que los procesos pueden incluir objetos (análogos al medio ambiente) sabemos que no existe límite. De un tiempo a esta parte, he preferido los procesos a los objetos, precisamente por esta razón: los procesos no excluyen a los objetos (Cage, 2007, pp. 162-163).

A pesar de los autores mencionados, es escasa la bibliografía en español de la enseñanza de la música contemporánea, sobre todo, a nivel universitario, aunque existen algunos estudios en la enseñanza secundaria, preludio de la enseñanza universitaria, Cureses (1998) o Urrutia y Díaz (2013), donde buena parte de la investigación, se desvía -como bien reconocen las autoras- hacia un tipo de música que resulta contemporánea, a nivel histórico, pero no estilítsico, a saber, la música popular urbana -pop, rock, etc.-, un campo musical extremadamente alejado de la estética de la música contemporánea. En efecto, en 1998, con un siglo XX que expiraba, Cureses afirmaba que "existen abundantes y bien conocidos precedentes en todos estos ámbitos que confirman la existencia de esa situación deficitaria en la educación y práctica musical contemporánea" (p. 215), mucho más, si consideramos, desde entonces, la disparidad de la asignatura de música en los sucesivos planes de estudios.

También han sido reconocidas las carencias de la didáctica de la música contemporánea en centros de mayor especialización musical que los de enseñanza secundaria, como universidades, conservatorios y escuelas de música, justo antes del fin del siglo XX, en que esta disciplina empezó a entrar en los planes de estudios a distintos niveles formativos:

Las dificultades derivadas de la situación inicial han constituido uno de los principales obstáculos para el desarrollo de una verdadera didáctica de la música contemporánea, tanto a niveles universitarios como en los centros profesionales y superiores de música - principalmente, en los Conservatorios y Escuelas musicales- y, por supuesto, en los Institutos de Enseñanza Secundaria (Cureses, 1998, p. 215).

Así ha defendido, por ejemplo, Tomás Marco, referente de la vanguardia en España, el proceso de enseñanza-aprendizaje de la música contemporánea y, como consecuencia de esta labor, la investigación sobre la misma a nivel musicológico, un estudio, por supuesto, que debe de partir de la enseñanza universitaria:

Un buen ejercicio para cualquier musicólogo, cualquiera que sea su especialidad temporal, sería ocuparse de tiempo en tiempo de temas recientes. Posiblemente, así la valoración de fatos referentes a pasados más lejanos cobraría una mayor óptica 
y, además, no se perdería la historia de la música como un proyecto evolutivo continuo, en el que cada momento no es un fin en sí mismo completamente cerrado como tantas veces parece desprenderse del análisis histórico (Marco, 1987, p. 399).

Dada la dificultad del modelo de enseñanza discipular propugnado por Stockhausen, en una época donde la enseñanza reglada, a todos los niveles, ha ocupado las cuotas educativas existentes, debemos trabajar por la integración y desarrollo de la música contemporánea en el aula universitaria, no sólo a través del plan de estudios, si no, también, desde el contenido y las estrategias didácticas -basadas en el análisis de procesos y estructuras- que nos permitan transmitir al alumnado una imagen holística de la música contemporánea, por un lado, y, por el otro, su inmediata conexión con los estilos del pasado, en pro de una enseñanza más creativa, abierta y curiosa, en un sentido estético:

En primer lugar, hay que ampliar nuestro horizonte en el campo de lo conocido: ver las obras, incluso, las obras maestras, en su movilidad; pensar más a menudo en lo que éstas abren en lo que logran, descubrir sobre un compositor, aunque el más aceptado, un punto de vista particularmente actual (...) esta escucha activa, esta participación real, si se trata del intérprete, nos lleva a ser más curiosos respecto del futuro (Boulez: 2008, p. 447).

De este modo, aun hoy, la presencia de la música contemporánea en la docencia a nivel superior es escasa o, incluso, está sometida a la elección del alumnado en asignaturas optativas que no implican, por tanto, el conocimiento del lenguaje contemporáneo en las nuevas promociones de nuevos músicos, de ahí que, en muchas ocasiones, como en el presente estudio, contemos con muestras de estudiantes muy reducidas.

Así pues, a continuación, vamos a presentar una investigación llevada a cabo con los alumnos de la asignatura Análisis de la música de los siglos XX y XXI en la Universidad Internacional de La Rioja (UNIR).

\section{Método}

\section{Objetivos}

- Objetivo 1: analizar las variables sociodemográficas de la muestra, a saber, género, edad, años de experiencia en la música, y su relación con los resultados de la asignatura.

- Objetivo 2: cuantificar los factores de aprendizaje, en función de la cuan- 
tía del trabajo realizado, así como el grado de conocimiento de los estudiantes por la materia dada.

- Objetivo 3: observar la naturaleza de los factores de aprendizaje y su interacción, así como las técnicas propias de la enseñanza on line, que han contribuido a los resultados de los estudiantes en la asignatura.

\section{Participantes}

Para este estudio contamos con una muestra aleatorizada, de doce alumnos de tercer curso de Grado de Música de la Universidad Internacional de La Rioja (UNIR), que se inscribieron, de manera voluntaria, en la asignatura optativa de segundo cuatrimestre Análisis de la música de los siglos XX y XXI. Todos los matriculados aceptaron participar en la investigación a través de un cuestionario, al final de la asignatura, durante el curso escolar 2016/2017.

Esta titulación, a la vista de su plan de estudios, ofrece un entorno favorable a la enseñanza de la música contemporánea en cuanto al número de materias ofertadas, desde distintos enfoques metodológicos, ya sean más de corte teórico -Historia de la música de los siglos XX y XXI-, sonológico y electro-acústico -Síntesis de sonido, Procesado de sonido, Composición electroacústica- y, finalmente, analítico y práctico -Análisis de la música de los siglos XX y XX, Notación de los siglos XX y XXI y Orquestación e Instrumentación contemporánea-. Sin embargo, algunas de estas asignaturas tienen un carácter optativo -las tres últimas mencionadas, por ejemplo-, lo cual no garantiza, desgraciadamente, una completa inmersión en la disciplina que nos ocupa, así como la utilización por necesidad de muestras de estudiantes cortas para las investigaciones.

Así pues, en el grupo $n=12$, se observan diversas variables socio-demográficas, género, edad, instrumento y años de experiencia en la música: $67 \%$ de hombres frente a $33 \%$ de mujeres; $33 \%$ de alumnos entre 18 y 30 años, $42 \%$ entre 31 y $40,17 \%$ entre 41 y $51,8 \%$ entre 51 y 60 ; amplia mayoría en cuanto a la práctica de la música durante diez años o más; especialidades instrumentales diversas, con un claro predominio del violín $(33 \%)$ y del canto $(17 \%)$, frente a otras opciones, como piano, clarinete, composición, percusión o guitarra, con un $8 \%$, respectivamente. 

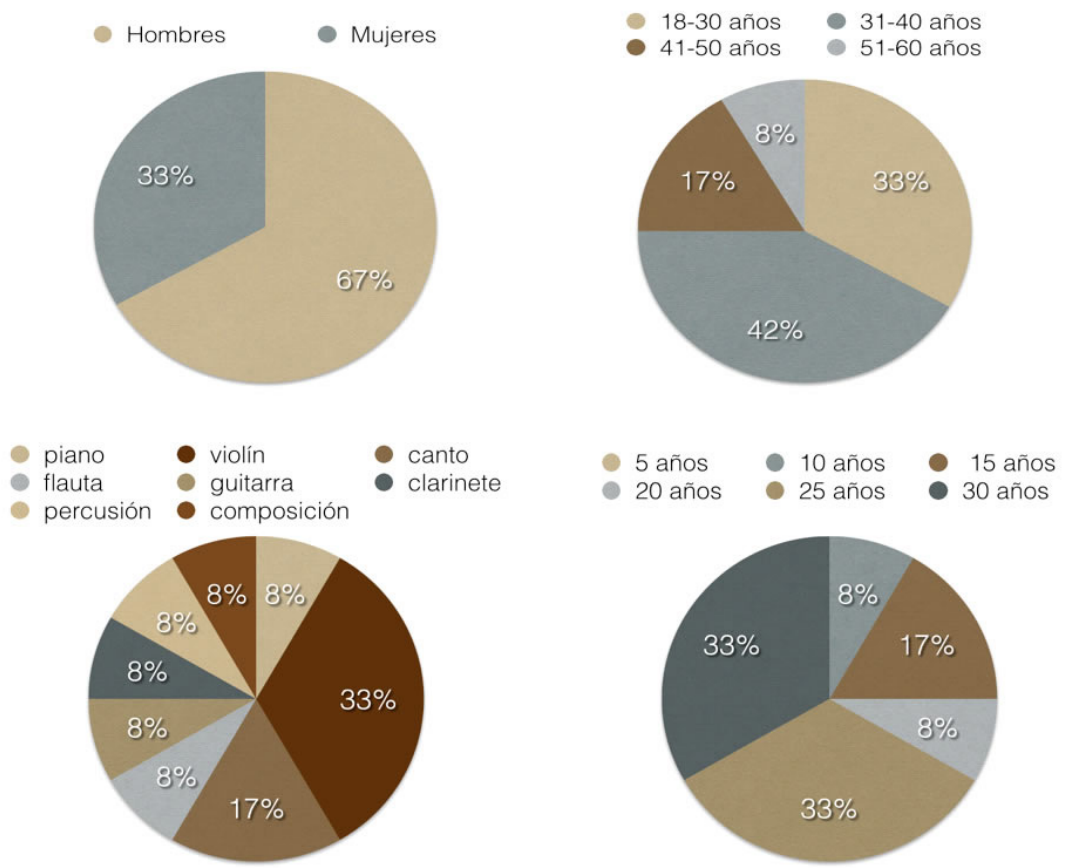

Figura 1. Gráficas de corte sociodemográfico, variables de género, edad, años de experiencia en la música y especialidad instrumental (elaboración propia).

Se ha considerado interesante reseñar que, a pesar de algunas nociones básicas, sobre todo, a nivel teórico -tras cursar alguna de las asignaturas obligatorias mencionadas anteriormente, como Historia de la música de los siglos XX y XXI-, ninguno de los sujetos participantes tenía experiencia previa en análisis y/o interpretación en el campo de la música contemporánea.

\section{Diseño}

Este estudio se ha enfocado como una correlación de variables personales y académicas que pretenden elucidar los factores de aprendizaje y el grado de conocimiento e interés por la materia, así como de aquellas estrategias proporcionadas por la enseñanza on line.

Por tanto, se plantea un diseño de investigación de tipo ex-post-facto o diseño selectivo, donde la distinción entre las variables independientes y dependientes es de tipo teórico. La recogida los datos, por medio de dos herramientas, un cuestionario on line -a través del dispositivo Google Drive- y la plataforma educativa de 
UNIR-en lo sucesivo, campus virtual-, ha tenido lugar en un solo momento, justo después de la finalización de la asignatura, en junio de 2017.

Por tanto, a causa del momento único de recogida de los datos, esta investigación se revela como un estudio de corte transversal.

\section{Instrumento}

Se ha elaborado un cuestionario ad hoc que recoge distintas variables sociodemográficas junto con datos referentes a la trayectoria académica y musical de los participantes, así como otras, de carácter puramente académico. Dicho cuestionario ha sido validado por tres jueces externos sobre su claridad, utilidad y pertinencia, de hecho, la subescala que mide el nivel de satisfacción (11 ítems; $\alpha$ de Cronbach $=.634)$ tiene un índice aceptable de consistencia interna.

Por otro lado, se han utilizado los datos registrados en el campus virtual de la Universidad Internacional de la Rioja, tanto el tiempo de conexión empleado para cada recurso de aprendizaje como las calificaciones de cada alumno.

Los datos han sido analizados mediante el software SPPS, Statistical Package for the Social Sciences de IMB.

A continuación, se presentan las variables de la investigación:

Variables académicas y personales: edad, género, instrumento, años de práctica en la música.

Un ítem tipo likert (0-10) para la valoración de la asignatura Análisis de la música de los siglos XX y XXI.

Datos acerca del tiempo empleado por los estudiantes a través de la plataforma, expresados en minutos: videoteca, ideas clave, anuncios, correo, documentación, estadísticas, foro, programa, tareas, videoteca, test y asistencia a clases en directo.

Datos sobre de los resultados de los estudiantes en la asignatura durante el curso 2016-2017: nota del examen, nota de la evaluación continua y nota final.

\section{Procedimiento}

La asignatura Análisis de la música de los siglos XX y XXI es optativa de tercer curso en el Grado de Música de UNIR - de cuatro años de duración y una carga lectiva de 240 créditos ECTS- y supone, por su parte, seis créditos, correspondientes a 180 horas de trabajo durante el cuatrimestre.

El contenido de la materia se divide en dos bloques, organizados cronológicamente, en seis temas cada uno: el primero, entre 1900 y 1945, donde se contemplan tendencias como Dodecafonismo, Neoclasicismo, Atonalidad, música 
popular etc., en cuanto al segundo, transcurre entre 1945 y la actualidad, y se compone de Serialismo integral, Música de textura, Espectralismo, Música aleatoria, concreta, electrónica y electroacústica, Minimalismo, Nueva Simplicidad, Nueva Complejidad y últimas tendencias.

La metodología empleada resulta 100\% on line, con clases semanales en directo, a través de videoconferencia que pueden verse en diferido si es imposible asistir en directo. Por otra parte, el campus virtual ofrece una amplia gama de recursos de aprendizaje, con los que el alumno puede trabajar los contenidos de la materia a su ritmo, según sus intereses, necesidades y capacidades, ideados para lograr un aprendizaje ágil, eficaz y autorregulado, como se puede observar en la Tabla 1:

\begin{tabular}{lc}
\hline Recurso & Tareas \\
\hline Ideas clave & Información básica (temas) \\
Anuncios & Alertas diarias, recordatorios, publicación de notas, cambios de clases, etc. \\
Correo & Sistema de mensajería interno con tutores, profesores y compañeros \\
Documentación & Material docente de refuerzo \\
Estadísticas & Consulta de notas \\
Foros & Recursos interactivos puntuables \\
Tareas & Instrucciones, fechas, procedimientos \\
Programación & Relación semanal de clases, actividades, lecturas \\
Test & Autoevaluación de conocimientos \\
Videoteca & Clases grabadas, clases magistrales \\
\hline
\end{tabular}

Tabla 1. Recursos de aprendizaje en el campus virtual.

En cuanto a la evaluación, se establece un porcentaje del 60\% para la nota del examen, frente a un $40 \%$ de la nota de la evaluación continua y otros recursos participativos de la plataforma, a saber, elaboración de trabajos (20\%), resolución de casos prácticos $(15 \%)$, participación en foros puntuables, test y asistencia a clases en directo $(5 \%)$, de este último porcentaje, cada test correcto cuenta 0.1 puntos y, cada clase en directo, otro 0.1 , pero sólo las dos primeras.

De este modo, la asignatura Análisis de la música de los siglos XX y XXI tuvo lugar entre la semana del 16 de marzo y la semana del 22 de junio de 2017, y transcurrió en los siguientes términos: se impartieron diecisiete sesiones presenciales de cuarenta y cinco minutos cada una, incluyendo catorce clases ordinarias, una de presentación, otra de dudas y otra de preparación del examen. Se plantearon, asimismo, cinco actividades, un foro puntuable, según la planificación de la Tabla 2, elaboradas bajo las coordenadas del análisis gráfico-sonoro: 


\begin{tabular}{lcc}
\hline Semana & Tareas & Autor/autores \\
\hline $24 / 3-27 / 3$ & Obra neoclásica (foro) & Ginastera \\
$3 / 4-7 / 4$ & Obras dodecafónicas & Schönberg, Berg, Webern \\
$17 / 4-21 / 4$ & Obra de entreguerras & Bartók \\
$1 / 4-5 / 4$ & Obra de posguerra & Messiaen \\
$15 / 4-19 / 4$ & Multiplicación de acordes & Boulez \\
$29 / 5-2 / 6$ & Música de textura & Ligueti, Xenakis \\
\hline
\end{tabular}

Tabla 2. Relación de trabajos, casos prácticos y foros puntuables.

El proceso de la investigación se ha elaborado a partir de la información registrada en la plataforma o campus virtual, en el transcurso de la asignatura, semana a semana, y, finalmente, en el cuestionario que fue respondido, una vez finalizada la asignatura, por la totalidad de la clase.

Aunque el programa de la asignatura, como se deduce de lo anterior, se caracteriza por nociones teóricas de cada una de las tendencias de la música del siglo XX -y, por extensión, la del XXI-, el contenido de la asignatura se centra en el análisis de la música del período elegido a partir de las premisas de Cage y Boulez en cuanto a las estructuras y procesos de un material sonoro centrado en el desarrollo de la textura y el timbre, y sus múltiples conexiones internas, como ha recordado Boulez, la música contemporánea debe ser "pensada en términos de relaciones, de funciones y no de sustancia y accidentes" (Boulez, 2009, p. 215).

Así pues, la parametrización tradicional en cuanto al lenguaje tonal de la música -estilo, forma, armonía, etc.-, deja paso a dos tipos de miradas analíticas, una visión global -con el transcurso del proceso- y una fragmentada -que da pie al estudio de las distintas estructuras que componen los procesos-, que son estudiadas en todos las tendencias mencionadas anteriormente, a partir del estudio de láminas gráfico-sonoras e, incluso, la elaboración de las mismas por parte del alumnado en las actividades propuestas a lo largo del curso.

Por otro lado, durante el transcurso de la asignatura se hace hincapié en las conexiones estilísticas y técnicas de la música de los siglos XX y XXI en cuanto a la parametrización de la música anterior a 1900 y el desarrollo de la misma hacia el concepto de procesos y estructuras, a modo de eslabón estético entre ambos bloques, como se observa en la figura 2: 

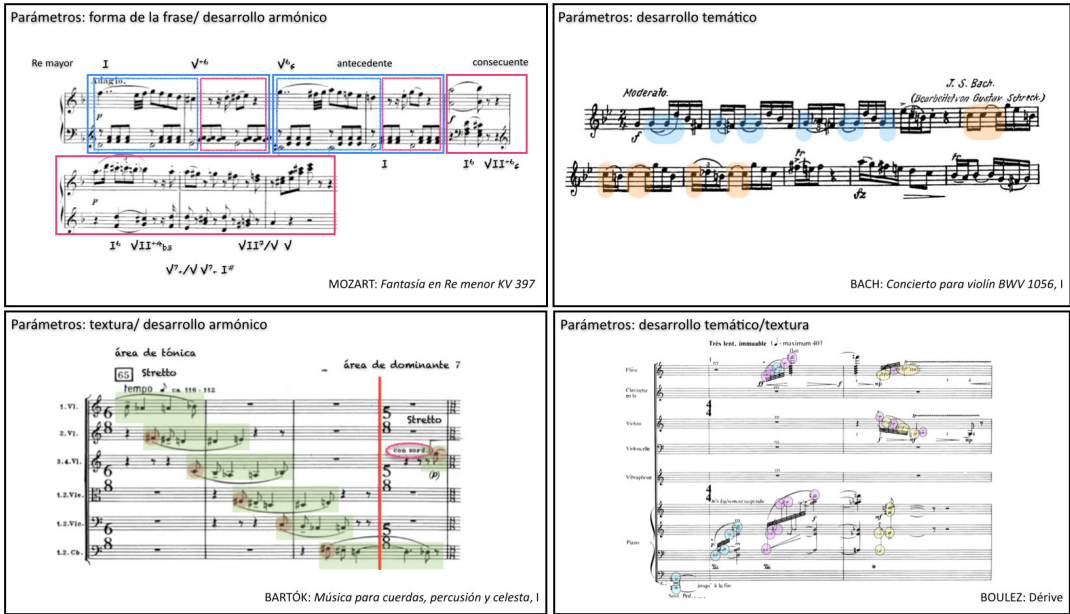

Figura 2: Láminas gráfico-sonoras comparativas entre el siglo XX y la música anterior a 1900.
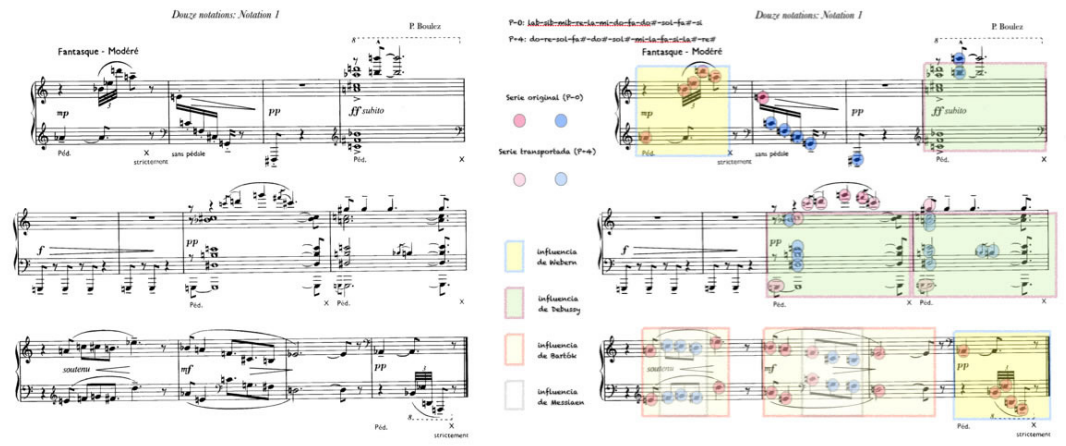

Figura 3: P. Boulez, Donz̨e Notations (1945), lámina original y lámina gráfico-sonora (elaboración propia).

En cuanto a la metodología, tanto en la exposición magistral de la clase como en la elaboración de las actividades exigidas en la evaluación continua, se ha utilizado la técnica del análisis gráfico-sonoro (Vela González, 2016; 2017; 2018) que plantea la profundización en la obra musical a partir de un estudio de parámetros globales y fragmentados sobre la partitura original, en cualquier época, manipulada a través de medios digitales, con el fin de reflejar diversas combinaciones paramétricas para el estudio de la obra y la mejora de la interpretación, con el objetivo 
de ofrecer distintas estrategias de estudio a los alumnos, en función de cada fase del estudio de la obra.

De este modo, la parametrización reducida a estructura y proceso cobra una nueva significación visual donde el análisis de la música contemporánea se centra en la propia percepción del alumnado, que se ejercita con la elaboración de sus propias láminas gráfico-sonoras a través de su progresiva experiencia en el análisis y, en efecto, de su capacidad de relación de cada ente sonoro en el conjunto.

\section{Resultados}

En cuanto a los resultados de los estudiantes en la mencionada asignatura, se han encontrado cuatro ítems cuantificables, la calificación del examen, la calificación de la evaluación continua, el tiempo empleado en la plataforma y la asistencia a clases en directo. Así pues, se ha obtenido un valor medio en el examen de 7,54 puntos -puntuación ponderada en un $60 \%$ de la nota final-; un valor medio de 3,72 puntos en la evaluación continua -sobre un total de cuatro, como $40 \%$ restante-, y una puntuación de 8,42 en cuanto a la media de la nota final.

En relación con la asistencia a clases en directo, los estudiantes han acudido a una media de 6,5 clases durante el cuatrimestre, lo que supone 390 minutos de dedicación; el porcentaje de alumnos ha sido de 4,58 por clase, con $n=12$.

Con respecto al tiempo apuntado en la plataforma, en el campus virtual se ha registrado una actividad media de 1684,58 minutos por alumno, es decir, aproximadamente, 28 horas al cuatrimestre, desglosado por recursos educativos según la Tabla 4:

A continuación, se ha elaborado un estudio de regresión lineal, a modo de diseño factorial, para conocer la ponderación de los ítems anteriores en el campus virtual. Esta prueba establece una variable dependiente, el tiempo del alumnado registrado en la plataforma (expresado en minutos), en relación con las variables denominadas como predictoras, que suponen el 85,23\% del tiempo total -ideas clave, videoteca, foros y anuncios-. Por su parte, la regresión lineal confirma el peso en el conjunto de cada variable predictora, en función de la variable dependiente, así pues, la variable de foros se muestra como el valor más fiable ( $\mathrm{p}=$ $.001 ; \beta=.401)$, seguida de la videoteca $(\mathrm{p}=.002 ; \beta=.583)$ y de las ideas clave $(\mathrm{p}=$ $.024 ; \beta=.360$ ), finalmente, la variable anuncios queda como la variable menos importante en la ponderación con una puntuación de $\mathrm{p}=.068$ y $\beta=.182$, superando levemente el umbral de la hipótesis nula. En este caso, el índice de correlación de Pearson asciende hasta el $r=.604 ; \mathrm{p}=.000$, un valor elevado. 
Epistemus - volumen 8 - número 1 (Julio de 2020)

DOI: https://doi.org/10.24215/18530494e018

\begin{tabular}{llcc}
\hline Fecha & $\mathrm{n}$ & Asistentes & Clase \\
\hline 16 de Marzo & 12 & 7 & Presentación \\
16 de Marzo & 12 & 7 & Ordinaria \\
22 de Marzo & 12 & 7 & Ordinaria \\
29 de Marzo & 12 & 4 & Ordinaria \\
7 de Abril & 12 & 8 & Ordinaria \\
10 de Abril & 12 & 6 & Ordinaria \\
19 de Abril & 12 & 5 & Ordinaria \\
20 de Abril & 12 & 5 & Ordinaria \\
3 de Mayo & 12 & 3 & Dudas \\
9 de Mayo & 12 & 2 & Ordinaria \\
12 de Mayo & 12 & 4 & Ordinaria \\
18 de Mayo & 12 & 5 & Ordinaria \\
24 de Mayo & 12 & 3 & Ordinaria \\
30 de Mayo & 12 & 4 & Ordinaria \\
7 de Junio & 12 & 3 & Ordinaria \\
15 de Junio & 12 & 1 & Ordinaria \\
22 de Junio & 12 & 4 & Examen \\
\hline
\end{tabular}

Tabla 3. Relación temporal de clases en directo y asistencia.

\begin{tabular}{lcc}
\cline { 2 - 3 } & $\mathrm{n}$ & Media $(\mathrm{min})$ \\
\hline Videoteca & 12 & 624,92 \\
\hline Ideas clave & 12 & 388,25 \\
Foro & 12 & 252,25 \\
Anuncios & 12 & 191,92 \\
Tareas & 12 & 90,00 \\
Documentación & 12 & 81,50 \\
Estadísticas & 12 & 68,58 \\
Test & 12 & 7,92 \\
Programación & 12 & 4,00 \\
Correo & 12 & 0,63 \\
\hline
\end{tabular}

Tabla 4. Relación de minutaje en recursos virtuales.

Por último, en un análisis correlacional de variables ( $r$ de Pearson) se observa una relación robusta entre la edad de los participantes y los años de práctica en 
la música $(\mathrm{r}=.735 ; \mathrm{p}=.006)$, así como una relación entre la nota final de la asignatura y la valoración de la misma $(\mathrm{r}=.013 ; \mathrm{p}=.691)$. Frente a una asociación lineal negativa, es decir, inversamente proporcional, entre el tiempo empleado en la plataforma y la calificación final de la asignatura ( $\mathrm{p}=.037$; $\mathrm{r}=-.607)$, se mantiene la hipótesis nula en el caso de la inexistente relación entre los respectivos valores de la evaluación continua y el examen $(\mathrm{r}=.152 ; \mathrm{p}=.637)$.

Se constata, también, que la variable de la asistencia a clases en directo no guarda relación con la variable de la calificación del examen ( $\mathrm{r}=-.171 ; \mathrm{p}=.595)$, ni con la de tiempo registrado en la plataforma $(\mathrm{r}=.379 ; \mathrm{p}=.224)$, ni tampoco con la de calificación de la evaluación continua $(\mathrm{r}=.222 ; \mathrm{p}=.489)$.

\section{Discusión}

En primer lugar, es necesario advertir que el estudio realizado ofrece ciertos problemas de generalización, dado el limitado número de sujetos participantes, que se traduce en una baja representatividad, debido, no obstante, a los criterios aleatorios de la selección de participantes en la investigación, o sea, la libre elección, por parte de los mismos, de la asignatura optativa Análisis de la música de los siglos XX y XXI, del segundo cuatrimestre del tercer curso del Grado de Música de la Universidad Internacional de La Rioja.

Sin embargo, los resultados obtenidos muestran el interés de los estudiantes hacia esta materia - un tanto árida, en la que carecían experiencia previa-, a partir de los cuatro factores de aprendizaje detectados, en los que cobra un papel determinante las estrategias de corte digital, propiciadas por la condición $100 \%$ on line de la enseñanza impartida.

En cuanto al primer objetivo, la muestra presenta una media de edad superior (en un 67\%), a los 31 años, a priori, una edad más infrecuente en estudios universitarios, por ejemplo, a la comprendida en el rango entre 18-31 años, al que pertenece el 33\% restante, proporción a la que, sin duda, contribuye la condición de la enseñanza a distancia. Del total de los participantes, $n=12$, se infiere, lógicamente, que, a mayor edad, más experiencia en la música, con una media bastante alta, de 23,7 años por cada alumno participante.

Por otra parte, la relación entre la valoración de la materia y la calificación final de la misma tiene un valor robusto $(r$ de Pearson $=691)$, atestiguando que, a mayor rendimiento en la asignatura, mejor opinión entre el alumnado. Además, se ha de recordar que la relación entre la calificación del examen y el tiempo empleado en la plataforma es inversamente proporcional, de ahí que la metodología empleada propicie que cada alumno construya su propio aprendizaje con los recursos de 
aprendizaje que considere convenientes según sus necesidades educativas individuales y/o sus conocimientos previos.

En cuanto al segundo objetivo, se observan cuatro factores principales en el aprendizaje de la materia, el examen y su calificación, la evaluación continua y su calificación -compuesta por cinco actividades, un foro puntuable y un pequeño porcentaje referido a la puntuación de los test y la asistencia de dos clases en directo-, las clases en directo y, por último, el tiempo invertido en el estudio del material complementario de la plataforma, a través de ocho ítems diferentes: videoteca, ideas clave, foros, anuncios, tareas, documentación, estadísticas, test, programación y correo. En los resultados individuales del alumnado, se observan calificaciones bastante elevadas, tanto en el examen, con una media de 7,54 sobre diez puntos, como en la evaluación continua, con una media de 3,72 sobre 4 puntos, de modo que la calificación final de la asignatura ronda el valor medio de 8,5 puntos.

Con respecto a la asistencia a clases en directo, se ha registrado una media de 6,5 clases por alumno a lo largo del cuatrimestre, advirtiendo que la asistencia no es obligatoria y que ofrece, como máximo, 0,2 puntos en la calificación de la evaluación continua, la media de asistencia ha sido de un $38,16 \%$, dato que habla acerca del interés de la asignatura por parte de los estudiantes.

Asimismo, se ha podido conocer la relación de horas empleadas en el estudio de la materia a través de la plataforma, con una media de 1684,58 minutos de media, es decir, aproximadamente, 28 horas por alumno, lo que supone un 15\% del tiempo contemplado en los 6 créditos ECTS estipulados en la asignatura; la media de asistencia en directo, por otra parte, supone 390 minutos de dedicación en el cuatrimestre, es decir, un 7\%. De este modo, el tiempo de dedicación virtual, entre clases en directo y recursos educativos, asciende a un $22 \%$ del total estipulado de la asignatura, al que debe sumarse el tiempo, imposible de determinar, que los estudiantes dedicaron al estudio del examen y a la elaboración de las láminas gráfico-sonoras demandadas en las actividades de la asignatura. De este modo, la plataforma y los recursos educativos virtuales disponibles se revelan como una herramienta sumamente útil a nivel formativo para el alumnado, dada la cantidad de tiempo utilizado en su uso, un $22 \%$ de media.

En cuanto al tercer objetivo, se ha constatado que los cuatro factores de aprendizaje observados - examen, evaluación continua, clases en directo y material complementario de la plataforma- no guardan relación entre sí, ni entre la nota de la evaluación continua y la nota del examen, ni entre la asistencia a las clases en directo con la calificación del examen y la evaluación continua, ni tampoco en cuanto al tiempo registrado en la plataforma. De este modo, se puede apreciar que los cuatro factores observados se erigen como compartimentos estancos que el 
estudiante puede abordar de manera independiente y que, frente a las directrices de la enseñanza tradicional en cuanto a la asistencia presencial a las clases, a partir de la metodología on line, se ofrece un modelo de enseñanza más flexible y autónomo, cuyo éxito no depende, como se aprecia en los resultados, de la percepción de la exposición magistral del profesor recibida -aun así, la asistencia en directo ha rondado casi el $40 \%$ de la clase-.

A través del campus virtual, se ha obtenido el dato de que los recursos de aprendizaje más utilizados han sido, por este orden, la videoteca, las ideas clave, los foros y los anuncios, mientras que los apartados de tareas, documentación y estadísticas consiguieron un minutaje mucho menor, muy alejado de los cuatro primeros ítems y que, por su parte, los últimos tres registros, test, programación y correo, obtuvieron un dato apenas residual en cuanto al tiempo empleado.

De esta forma, los cuatro primeros ítems suman un $85,23 \%$ por ciento del tiempo total, frente al 14,77 restante y, por su parte, el análisis de regresión indica que, dentro de los ítems con mayor puntuación, la ponderación del tiempo empleado ordena los valores como se puede observar en la Tabla 5.

\begin{tabular}{lccc}
\cline { 2 - 4 } & Beta & Sig. & $\%$ \\
\hline Foros & 0,401 & 0,001 & 14,75 \\
Videoteca & 0,583 & 0,002 & 36,55 \\
Ideas clave & 0,360 & 0,024 & 22,71 \\
Anuncios & 0,182 & 0,068 & 11,22 \\
\hline
\end{tabular}

Tabla 5: Relación en recursos virtuales predominantes.

Este dato arroja la conclusión de que los alumnos han preferido los recursos educativos del foro puntuable y de la videoteca, que contiene clases grabadas, para ver en diferido, como clases magistrales, es decir, clases grabadas con material de refuerzo, antes que otros recursos, también puntuables, como la autoevaluación de conocimiento a través del test.

De este modo, los materiales de corte virtual de la asignatura, esto es, las clases en directo por vídeo conferencia y los recursos educativos del campus virtual, han irrumpido con fuerza en el tiempo estipulado por los 6 créditos ECTS, hasta un $22 \%$ del minutaje total registrado, mientras que otros ítems, preparación del examen y elaboración de las actividades, han sido imposibles de cuantificar en las 180 horas contempladas.

Por tanto, del presente estudio pueden desprenderse las conclusiones: 
- La enseñanza de la música contemporánea puede despertar el interés del alumnado a nivel universitario sin experiencia previa, lo cual se traduce en unas elevadas calificaciones y en una asistencia, asimismo alta, desde un modelo de enseñanza on line que no exige, per se, la asistencia a clases en directo.

- Se han detectado cuatro factores incidentes en el aprendizaje de la materia, examen, evaluación continua, recursos de la plataforma y clases en directo, completamente independientes entre ellos, lo que condiciona el éxito de la asignatura a distintas causas, más allá de lo esperable en la enseñanza de tipo presencial, basada, fundamentalmente, en la asistencia a clase y en la calificación del examen.

- La metodología de enseñanza on line posibilita una serie de recursos educativos que propician no sólo el enriquecimiento del contenido de la materia, sino, también, la libre elección de estos recursos durante el proceso de enseñanza-aprendizaje y su autorregulación por parte de los estudiantes, en función de sus necesidades individuales y de sus conocimientos previos.

- La puesta en práctica de técnicas didácticas digitales, como la utilización del análisis gráfico-sonoro, puede ayudar al estudiante sin experiencia previa en música contemporánea a iniciarse con éxito en este ámbito, desde de las mismas técnicas analíticas que exige el repertorio musical anterior al siglo XX.

- La música contemporánea debe ser una materia inalienable en los estudios musicales de nivel superior, dentro del bloque de análisis musical, como punto de encuentro entre el pasado más inmediato y el futuro de nuestro arte.

Así pues, los resultados de la asignatura muestran una influencia positiva en la enseñanza de la música contemporánea, no sólo en cuanto al interés del alumnado -que invirtió el 22\% del tiempo estipulado por la configuración de créditos en el estudio de los recursos de la plataforma, dedicados a la inmersión en la materia, a partir de vídeos, artículos, test, clases grabadas y etc.,-, si no, también, a las propias calificaciones de los estudiantes matriculados $-7,54$ sobre 10 en el examen final; 3,72 sobre 4 en la puntuación de las actividades-, en una asignatura a la que acudían con una base de conocimientos previos en análisis e interpretación de música contemporánea sumamente limitada o inexistente.

De este modo, a través de una variada gama de recursos educativos relacionados con la enseñanza on line y, por tanto, con las nuevas tecnologías, es posible obtener buenos resultados académicos en un área, de la de música contemporá- 
nea, tal vez, árdua para los estudiantes, a causa de su todavía hoy escasa presencia en los planes de estudios -situación que esperamos empiece a cambiar con el paso del tiempo-, partiendo de un modelo analítico basado en una nueva parametrización, basado en procesos y estructuras, que acentúe la comprensión de los lenguajes contemporáneos hasta la actualidad:

"Ciertamente, sentir placer con la música es la señal de comprensión más obvia, la prueba de que la entendemos, un sentimiento en que podemos incluir la afinidad que experimentamos con los oyentes que también deleita...” (Rosen, 2017, p. 11).

\section{Notas}

1. Marta Vela González: https://orcid.org/0000-0002-5700-6767

2. Boulez murió el 5 de enero de 2016. En 1951 publicó Schönberg est mort, un artículo donde criticaba fieramente la estética schönbergiana por su acercamiento a la música posromántica del último tercio del siglo XIX, sin embargo, ninguna de las voces actuales de la música contemporánea se ha atrevido a publicar un Boulez est mort.

\section{Referencias}

Adorno, T. W. (2000). Sobre la música. Barcelona: Paidós.

Adorno, T. W. (1990). Berg. Alianza, Madrid: Alianza.

Ansermet, E. (2000). Escritos sobre música. Barcelona: Idea Música.

Buch, E. (2010). El caso Schönberg. Buenos Aires: Fondo de Cultura Económica.

Boulez, P. (2009). Pensar la música hoy. Murcia: Colegio oficial de aparejadores y arquitectos de la Región de Murcia.

Boulez, P. (2008). Puntos de referencia. Barcelona: Gedisa.

Boulez, P. (2002). La escritura del gesto. Barcelona: Gedisa.

Cage, J. (2007). Escritos al oído. Murcia: Colegio oficial de aparejadores y arquitectos de la Región de Murcia.

Corredor, J. (1985). Casals. Barcelona: Salvat.

Cureses de la Vega, M. (1998). La música contemporánea en la educación secundaria. Aula Abierta n ${ }^{\circ}$ 71, pp. 213-235. 
Epistemus - volumen 8 - número 1 (Julio de 2020)

DOI: https://doi.org/10.24215/18530494e018

Dibelius, U. (2005). La música contemporánea a partir de 1945. Madrid: Akal.

Furtwängler, W. (2011). Conversaciones sobre música. Barcelona: Acantilado.

Griffiths, P. (2010). Modern music and after. Oxford: Oxford University Press.

Harnoncourt, N. (2016). Diálogos sobre Mozart. Barcelona: Acantilado.

Harnoncourt, N. (2006). La música como discurso sonoro. Barcelona: Acantilado.

Hemsy de Gainza, V. (2002). Pedagogía musical. Dos décadas de pensamiento y acción educativa. Buenos Aires: Lumen.

Lagrange, H. L. (2002). Viena, una historia musical. Barcelona: Paidós.

Madigan, M. (2008). Un acto de libertad. Charlando con Antoni Ros-Marbà. Madrid: Ediciones Autor.

Mantel, G. (2010). Interpretación. Del texto al sonido. Madrid: Alianza.

Marco, T. (1987). Los años cuarenta España en la música de occidente: actas del congreso internacional celebrado en Salamanca, 29 de octubre-5 de noviembre de 1985, "Año Europeo de la Música" / coord. por José López-Calo, Ismael Fernández de la Cuesta, Emilio Francisco Casares Rodicio, Vol. 2, pp. 399-412.

Marín, M. A. (2018). El 'big data’ prueba el conservadurismo en la música clásica en vivo. Extraído el 30 de julio de 2018 desde https://elpais.com/cultura/2018/07/22/actualidad/1532252915_160718.html

Menhuin, Y. (2005). Lecciones de vida. Barcelona: Gedisa.

Rosen, C. (2017). Las fronteras del significado. Barcelona: Acantilado.

Rosen, C. (2014). El piano: notas y vivencias. Madrid: Alianza.

Ross, A. (2010). El ruido eterno. Barcelona: Seix Barral.

Schoenberg, A. (1988). Cartas. Madrid: Turner.

Stockhausen, K. (1988) Entrevista sobre el genio musical. Madrid: Turner.

Swarowsky, H. (1989). Defensa de la obra. Madrid: Real Musical.

Toch, E. (2001). Elementos constitutivos de la música. Barcelona: Idea Books.

Urrutia, A., y Díaz, M. (2013). La música contemporánea en la educación secundaria: características, prácticas docentes y posicionamiento del profesorado. Revista de Investigación Educativa ${ }^{\circ}$ 17, pp. 1-40. 
Vela González, M. (2018). El análisis gráfico-sonoro como fuente de conocimiento de la obra musical en estudiantes de la Universidad Internacional de La Rioja. REDU - Revista de Docencia Universitaria, 16 (2), pp. 231-247. https://doi.org/10.4995/ redu Recuperado de https://polipapers.upv.es/index.php/REDU/article/ view/8837/10947

Vela González, M. (2018). Metodología de análisis gráfico-sonoro: estudio paramétrico de la obra en alumnos del Grado de Música de UNIR. Revista Neuma, Año 10, Vol. 2, Universidad de Talca. Recuperado de http:// neuma.utalca.cl/?p=210

Vela González, M. (2016). Análisis gráfico-sonoro en la enseñanza de la interpretación musical. UNIR Revista. Recuperado de https://www.unir.net/humanidades/revista/noticias/analisis-grafico-sonoro-en-la-ensenanza-de-la-interpretacion-musi$\mathrm{cal} / 549201507961 /$

Webern, A. (2009). El camino de la nueva música. Barcelona: Nortesur.

Walter, B. (2002). Gustav Mabler. Madrid: Alianza.

Xenakis, I. (1955). La crise de la musique sérielle. Gravesaner Blätter ${ }^{\circ}{ }^{\circ}$, pp. 2-4. 